\title{
Prevalence of SARS-CoV-2 Antibodies after First 6 Months of COVID-19 Pandemic, Portugal
}

Luísa Canto e Castro, Ana Helena Guia Pereira, Rita Ribeiro, Catarina Alves, Luís Veloso, Vera Vicente, Dalila Alves, Inês Domingues, Cláudia Silva, Andreia Gomes, Marta Serrano, Ângela Afonso, Marc Veldhoen, Maria José Rego de Sousa, José Germano Rego de Sousa, Germano de Sousa, Maria M. Mota, Bruno Silva-Santos, ${ }^{1}$ Ruy M. Ribeiro ${ }^{1}$

In September 2020, we tested 13,398 persons in Portugal for antibodies against severe acute respiratory syndrome coronavirus 2 by using a quota sample stratified by age and population density. We found a seroprevalence of $2.2 \%, 3-4$ times larger than the official number of cases at the end of the first wave of the pandemic.

Cevere acute respiratory syndrome coronavirus S2 (SARS-CoV-2) has spread rapidly worldwide during 2020-2021, but incidence has been highly variable in different countries and is difficult to estimate. In Portugal, which has $\approx 10.3$ million inhabitants, the burden of disease, cases, and deaths was similar to or less than that for neighboring countries during the first wave of the coronavirus disease (COVID-19) pandemic, through September 2020 (Appendix Figure, https://wwwnc.cdc.gov/ EID/article/27/11/21-0636-App1.pdf). However, it is difficult to estimate the true extent of SARSCoV-2 infections in Portugal, although a previous study of clinical patients indicated a seropositivity $\leq 2.9 \%$ (1). We report a national, cross-sectional, epidemiologic survey that used quota sampling to quantify more accurately the cumulative number of infected persons in Portugal.

Author affiliations: Fundação Francisco Manuel dos Santos, Lisbon, Portugal (L. Canto e Castro); Universidade de Lisboa, Lisbon

(L. Canto e Castro, I. Domingues, C. Silva, A. Gomes, M. Serrano,

Â. Afonso, M. Veldhoen, M.M. Mota, B. Silva-Santos, R.M. Ribeiro);

Centro de Medicina Laboratorial Germano de Sousa, Lisbon

(A.H.G. Pereira, R. Ribeiro, M.J. Rego de Sousa, J.G. Rego de Sousa, G. de Sousa); CTI Clinical Trial and Consulting Services, Portugal (C. Alves, L. Veloso, V. Vicente, D. Alves); Los Alamos National Laboratory, Los Alamos, New Mexico, USA (R.M. Ribeiro)

DOI: https://doi.org/10.3201/eid2711.210636

\section{The Study}

We used a convenience quota sampling, quasi-proportional to the population of Portugal in 9 strata: age group $(<18,18-54$, and $\geq 55$ years of age), each subdivided by population density of place of residence $\left(<60,60-500\right.$, and $>500$ persons $\left./ \mathrm{km}^{2}\right)$ (Appendix). After a widespread media campaign, we recruited participants by using voluntary registration on a website specifically designed for this study. We obtained informed consent from all participants $\geq 16$ years of age and from legal guardians for participants $<18$ years of age. The study was approved by the Ethics Committee of the Centro Académico de Medicina de Lisboa (\#350/20, July 30, 2020).

Blood collections and serologic tests were performed by Centro de Medicina Laboratorial Germano de Sousa (Lisbon, Portugal) by using standard procedures. We determined total antibodies against SARS-CoV-2 by using a chemiluminescent immunoassay test (COV2T; Advia Centaur Siemens, https:/ / www.siemens-healthineers.com), which targets the spike protein. This antibody test has a sensitivity of $98.1 \%$ and a specificity of $99.9 \%$ (2), which we used to correct the seroprevalence estimates by using the Rogan-Gladen estimator (3). We used sample weights and poststratified by sex to adjust the seroprevalence, extrapolating from the strata to the whole population (Appendix Tables 1-4). Participants completed a questionnaire with demographic, clinical, and epidemiologic questions regarding SARS-CoV-2 exposure (Appendix). We use standard statistical analyses to compare results at an $a=0.05$ significance.

We enrolled 13,398 participants $(55.3 \%$ women, age range 1-92 years) (Appendix Figure 2). Our sample reflected approximately the characteristics of the

${ }^{1}$ These authors were co-senior authors. 
Table 1. Prevalence of antibodies against severe acute respiratory syndrome coronavirus 2 , by person age, adjusted for sensitivity and specificity, Portugal, September 8-October 14, 2020

\begin{tabular}{lcccc}
\hline & \multicolumn{4}{c}{ Seroprevalence, \% $(95 \% \mathrm{Cl})$, by age, $\mathrm{y}$} \\
\cline { 2 - 5 } Population density & $<18, \mathrm{n}=2,108$ & $18-54, \mathrm{n}=6,495$ & $\geq 55, \mathrm{n}=4,795$ & Overall, $\mathrm{n}=13,398$ \\
\hline Low, $\mathrm{n}=2,298$ & $0.6(0.2-2.8)$ & $1.5(0.9-2.6)$ & $1.7(1.0-2.9)$ & $1.4(1.1-2.2)$ \\
Medium, $\mathrm{n}=5,006$ & $1.4(0.8-2.7)$ & $1.7(1.3-2.4)$ & $1.7(1.2-2.5)$ & $1.6(1.4-2.1)$ \\
High, $\mathrm{n}=6,094$ & $3.5(2.5-5.0)$ & $3.1(2.6-3.9)$ & $2.2(1.7-3.1)$ & $2.9(2.5-3.4)$ \\
Overall & $2.4(1.9-3.3)$ & $2.3(2.0-2.8)$ & $1.9(1.6-2.4)$ & $2.2(2.0-2.5)$ \\
\hline
\end{tabular}

population in Portugal, except for overrepresentation of women, persons who had higher levels of education, persons living in households that had $>1$ person, and workers in the education and health sectors (Appendix Tables 5-7).

We obtained blood samples during September 8 -October 14, 2020; a total of $\approx 90 \%$ were obtained by September 19. Overall seroprevalence was $2.2 \%(95 \%$ CI $2.0 \%-2.5 \% ; \mathrm{n}=296$ positive participants) (Table $1)$. The differences seen among age groups did not reach statistical significance. We found a higher seroprevalence in regions of high population density $(2.9 \%, 95 \%$ CI $2.5 \%-3.4 \%)$ versus regions of medium population density $(1.6 \%, 95 \%$ CI $1.4 \%-2.1 \%)$ and low population density $(1.4 \%, 95 \%$ CI $1.1 \%-2.2 \%)$ (Appendix Figure 3).

Comparing the seroprevalence $(2.2 \%$ corresponds to $\approx 226,000$ persons in Portugal) with the number of official cumulative confirmed cases $(55,720$ on August 24 and 76,396 on October 1) (4), we found a 3-4-fold larger number of persons who had antibodies than those reported infected. This factor varied across age groups; we found an $\approx 9$-fold difference for young participants versus a 2-5-fold difference (depending on sex and age) in middle-age and older participants (Figure 1). With our estimate of cumulative cases, we calculated that the infection-fatality rate varied from $\leq 0.2 \%$ in younger persons to up to $9.0 \%$ in men $>80$ years of age (Figure 2). The estimated proportion of asymptomatic persons among seropositive persons was $17.4 \%$ (95\% CI $14.1 \%-22.9 \%)$; this proportion was much higher for persons $<18$ years of age (Appendix Table 8).

We found no difference between seropositivity levels in men and women $(2.3 \%$ vs. $2.1 \%)$ (Table 2; Appendix Table 9). There were small differences in seroprevalence by occupation and professional sector; and teleworkers had a lower seroprevalence $(1.4 \%)$ than nonteleworkers (2.4\%) (Table 2). We also did not find differences in seroprevalence for persons who had chronic conditions versus persons who did not (Appendix Table 10). One of the largest differences was between nonsmokers and smokers $(2.4 \%$ vs. $1.0 \%$ ) (Table 2 ).

Of the seropositive participants, $50.0 \%$ had never been given a diagnosis as being a case or a suspected case of infection (Appendix Table 11). However, $5 \%(n=669)$ of participants were considered as having a suspected case of COVID-19 before the study (Table 2). This number is consistent with the number of suspected cases, which the national health authorities reported until August 16, 2020, two weeks before the start of our study, when there were a cumulative 468,937 suspected cases (only 54,102 confirmed), corresponding to $4.6 \%$ of the population of Portugal.

\section{Conclusions}

We found a seroprevalence of $2.2 \%$ for antibodies against SARS-CoV-2 in the population of Portugal,
Figure 1. Seroprevalence of antibodies against severe acute respiratory syndrome coronavirus 2, Portugal, compared with official reported confirmed cases, by sex and age. A) Female; B) male. Adjusted seroprevalence measured in this study (numbers above light gray bars) is compared with confirmed cases (numbers above dark gray bars) as a fraction of the corresponding population group (on September 1, 2020). Error bars indicate $95 \% \mathrm{Cls}$ for estimates. This figure includes different age ranges for consistency with the official data on number of cases by age.

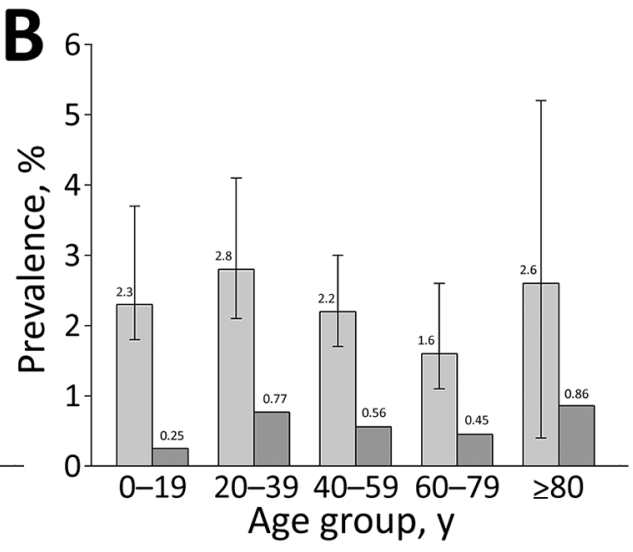




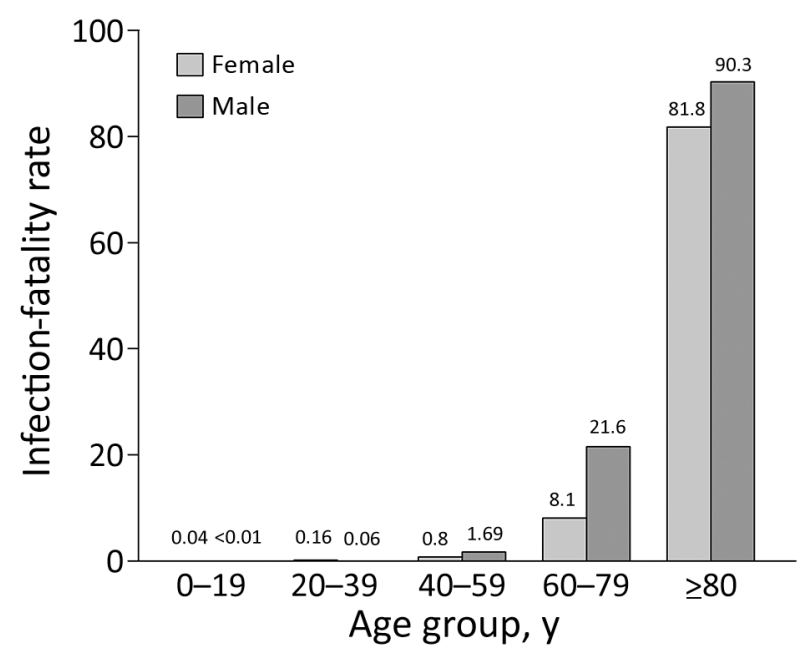

Figure 2. Inferred infection-fatality rate from seroprevalence estimates for antibodies against severe acute respiratory syndrome coronavirus 2, Portugal. We used the registered number of deaths (on September 21, 2020) by age and sex and our prevalence estimates based on seropositivity to infer the infection-fatality rate (Appendix, https://wwwnc.cdc.gov/EID/ article/27/11/21-0636-App1.pdf) for more details. Numbers above bars indicate deaths per 1,000 persons.

which was lower than that in a previous smaller study (1). Our results suggest that 3-4-fold as many persons were infected by SARS-CoV-2 than was officially reported by health authorities. This factor is consistent with, albeit somewhat smaller than, results reported in other national seroprevalence studies (57) and varied across age groups.

The higher seroprevalence in younger participants is in contrast to the official number of confirmed cases in Portugal, where there is a higher prevalence in older persons (4), possibly because younger persons tend to have milder disease, often asymptomatic $(8,9)$. We found that $\approx 40 \%$ of infections were asymptomatic in persons $<18$ years of age, whereas this proportion was much lower in older persons. Overall, if only $\approx 20 \%$ of cases are asymptomatic, a question is why so many cases go undetected even with higher testing rates, as in Portugal before our study (10). This discrepancy highlights the public health relevance of conducting seroprevalence studies.

Despite a similar prevalence, we found that the infection-fatality rate for men was higher than that for women, particularly in persons $>40$ years of age. The rate was more than twice as large for persons $60-79$ years of age $(2.16 \%)$ than for the overall group $(0.81 \%)$. These values are consistent with those reported in Spain (11) and include only confirmed COVID-19 deaths, not all excess deaths during this period (12).
A limitation of our study is that we used quota sampling, relying on volunteers for the study. We chose our method of recruitment to achieve a fast enrollment process because, during a pandemic, the number of persons positive for antibodies is changing continuously. We reasoned that such changes could bias the study more than the method of recruitment. In addition, even studies with a fully random sample often have a large fraction of persons refusing to participate or unable to be contacted $(6,13)$. Another limitation is that we used relatively large intervals for age groups. A more fine-grained stratification, along with other variables (e.g., sex), would be more representative of epidemiologic and clinical aspects of SARS-CoV-2, but would require a larger sample. We also did not correct for potential seroreversion (14), which reduces the fraction of seropositive results in relation to the actual number of infections and lowers the estimated infection-fatality rate. However, we expect seroreversion over the short 6-month period covered by our study to be minimal (15). These potential limitations are common to most seroprevalence studies but do not limit the need for conducting these studies during the evolving pandemic.

Overall, our results demonstrate a low incidence of SARS-CoV-2 during the first wave (spring and summer 2020) of the pandemic in Portugal. This incidence probably resulted from control measures that were relatively successful, in comparison with other countries with higher seroprevalence over similar (or shorter) periods (6).

\section{Acknowledgments}

We thank the 13,398 volunteers for participating in this study; colleagues at Universidade de Lisboa, Centro de Medicina Laboratorial Germano de Sousa, and CTI Clinical Trial and Consulting Services, in particular Filipa Robalo, Liliana Cunha, and Luis Graça for providing help and assistance; and the 5 anonymous reviewers, whose comments substantially improved the manuscript.

This study was supported by Sociedade Francisco Manuel dos Santos and Grupo Jerónimo Martins. Portions of this study were supported by the LANL LDRD Program (grant 20210730ER), Fundacao para a Ciencia e Tecnologia (Portugal) (grants PTDC/MAT-APL/31602/2017 and UID/MAT/00006/2019), and the European Union Horizon 2020 Research and Innovation Program (grant no. No 952377: iSTARS ERA Chair). M.V. was supported by the European Union H2020 ERA project (grant 667824: EXCELLtoINNOV). 


\section{About the Author}

Dr. Canto e Castro is professor of statistics at the Faculdade de Ciências da Universidade de Lisboa, Lisbon, Portugal. Her primary research interests are biostatistics; extreme value theory; and collection, analysis, and dissemination of statistical information.

\section{References}

1. Kislaya I, Gonçalves P, Barreto M, Sousa R, Garcia AC, Matos R, et al.; ISNCOVID-19 Group. Seroprevalence of SARS-CoV-2 infection in Portugal in May-July 2020: results of the first National Serological Survey (ISNCOVID-19). Acta Med Port. 2021;34:87-94. https://doi.org/10.20344/ amp.15122

2. Ainsworth M, Andersson M, Auckland K, Baillie JK, Barnes E, Beer S, et al.; National SARS-CoV-2 Serology Assay Evaluation Group. Performance characteristics of five immunoassays for SARS-CoV-2: a head-to-head benchmark comparison. Lancet Infect Dis. 2020;20:1390-400. https:/ / doi.org/10.1016/S1473-3099(20)30634-4

3. Rogan WJ, Gladen B. Estimating prevalence from the results of a screening test. Am J Epidemiol. 1978;107:71-6. https:/ / doi.org/10.1093/oxfordjournals.aje.a112510

4. Portuguese Ministry of Health. Status Report COVID-19 [in Portuguese] [cited 2021 May 14]. https:/ / covid19.min-saude. $\mathrm{pt} /$ relatorio-de-situacao

5. Gudbjartsson DF, Norddahl GL, Melsted P, Gunnarsdottir K, Holm H, Eythorsson E, et al. Humoral immune response to SARS-CoV-2 in Iceland. N Engl J Med. 2020;383:1724-34. https://doi.org/10.1056/NEJMoa2026116

6. Pollán M, Pérez-Gómez B, Pastor-Barriuso R, Oteo J, Hernán MA, Pérez-Olmeda M, et al.; ENE-COVID Study Group. Prevalence of SARS-CoV-2 in Spain (ENE-COVID): a nationwide, population-based seroepidemiological study. Lancet. 2020;396:535-44. https://doi.org/10.1016/ S0140-6736(20)31483-5

7. Poustchi H, Darvishian M, Mohammadi Z, Shayanrad A, Delavari A, Bahadorimonfared A, et al. SARS-CoV-2 antibody seroprevalence in the general population and high-risk occupational groups across 18 cities in Iran: a population-based cross-sectional study. Lancet Infect Dis. 2020.
8. Beale S, Hayward A, Shallcross L, Aldridge RW, Fragaszy E. A rapid review and meta-analysis of the asymptomatic proportion of PCR-confirmed SARS-CoV-2 infections in community settings. Wellcome Open Res. 2020;5:266 [cited 2021 Aug 16]. https:/ / doi.org/10.12688/wellcomeopenres.16387.1

9. Syangtan G, Bista S, Dawadi P, Rayamajhee B, Shrestha LB, Tuladhar R, et al. Asymptomatic SARS-CoV-2 carriers: a systematic review and meta-analysis. Front Public Health. 2021;8:587374. https:// doi.org/10.3389/fpubh.2020.587374

10. Triunfol M. High COVID-19 testing rate in Portugal. Lancet Infect Dis. 2020;20:783. https:/ / doi.org/10.1016/ S1473-3099(20)30499-0

11. Pastor-Barriuso R, Pérez-Gómez B, Hernán MA, Pérez-Olmeda M, Yotti R, Oteo-Iglesias J, et al.; ENE-COVID Study Group. Infection fatality risk for SARS-CoV-2 in community dwelling population of Spain: nationwide seroepidemiological study. BMJ. 2020;371:m4509. https:// doi.org/10.1136/bmj.m4509

12. Nogueira PJ, Nobre MA, Nicola PJ, Furtado C, Vaz Carneiro A. Excess mortality estimation during the COVID-19 pandemic: preliminary data from Portugal. Acta Med Port. 2020;33:376-83. https:/ / doi.org/10.20344/ amp.13928

13. Hallal PC, Hartwig FP, Horta BL, Silveira MF, Struchiner CJ, Vidaletti LP, et al. SARS-CoV-2 antibody prevalence in Brazil: results from two successive nationwide serological household surveys. Lancet Glob Health. 2020;8:e1390-8. https:/ / doi.org/10.1016/S2214-109X(20)30387-9

14. Shioda K, Lau MS, Kraay AN, Nelson KN, Siegler AJ, Sullivan PS, et al. Estimating the cumulative incidence of SARS-CoV-2 infection and the infection fatality ratio in light of waning antibodies. Epidemiology. 2021;32:518-24. https://doi.org/10.1097/EDE.0000000000001361

15. Figueiredo-Campos P, Blankenhaus B, Mota C, Gomes A, Serrano M, Ariotti S, et al. Seroprevalence of anti-SARS-CoV-2 antibodies in COVID-19 patients and healthy volunteers up to 6 months post disease onset. Eur J Immunol. 2020;50:2025-40. https://doi.org/10.1002/ eji.202048970

Address for correspondence: Ruy M. Ribeiro, Department of Theoretical Biology and Biophysics, Los Alamos National Laboratory, Mailstop K710, Los Alamos, NM 87545, USA; email: ruy@lanl.gov 